N⿳91-10956

\title{
GRAPHICAL INTERFACES FOR COOPERATIVE PLANNING SYSTEMS
}

\section{Philip J. Smith and Chuck Layton Ohio State University}

\author{
and
}

\section{Elaine McCoy}

San Jose State University 
$:$
$\vdots$
$\vdots$
$\vdots$
$\vdots$
$\vdots$
$\vdots$
$\vdots$
$\vdots$
$\vdots$
$\vdots$
$\vdots$
$\vdots$
$\vdots$
$\vdots$
$\vdots$
$\vdots$
$\vdots$
$\vdots$
$\vdots$
$\vdots$
$\vdots$ 


\begin{abstract}
Based on a cognitive task analysis of 5 airline flight crews in a simulator study, we have designed a testbed for studying computer aids for en route flight path planning. This testbed runs on a Mac II controlling three color monitors, and is being used to study the design of aids for both dispatchers and flight crews.

Specifically, our research focuses on design concepts for developing cooperative problem-solving systems. We use en route flight planning (selecting alternate routes or destinations due to unanticipated weather, traffic, malfunctions, etc.) as the context for studying the design of such systems. Flight planning provides an interesting context because
\end{abstract}

1. Decisions must be made based on multiple competing or complementary goals.

2. Decisions are made in an information-rich environment.

3. Some of the information is available only to the flight crew (e.g., visual data or verbal reports from other planes and air traffic control). Other information is most easily accessed or processed by the computer.

4. Decisions must be made in a stochastic world. There is a great deal of uncertainty about future events.

5. There is the potential to apply both knowledge-based systems and optimization approaches in the design of computer aids.

6. Much (but not all) of the data is very graphic in nature.

We are currently exploring three questions in this test environment:

1. When interacting with a flight planning aid, how does the role of the pilot influence overall system performance? (Should the computer aid generate and recommend full flight plans; and should it respond to "what if" explorations by the pilot, etc.?)

2. Can the architecture for a cooperative planning system be built around Sacerdoti's (1983) concept of an abstraction hierarchy, where the pilot can interact with the system at many different levels of detail (but where the computer aid by default handles lower level details that the pilot has chosen not to deal with)?

3. Can graphical displays and direct manipulation of these displays provide perceptual enhancements (Larkin and Simon, 1987) of the pilot's problem-solving activities? 


\section{Motivation}

Use "aiding/automation only at those points in time when human performance in a system needs support to meet operational requirements - in the absence of such needs, human performance remains unaided/manual, and thereby humans remain very much "in the loop", (Rouse, 1988).

"Users will not accept an aiding system that appears to usurp their authority or unduly restricts their options", (Madni, 1988).

"The improvement of cooperative problem solving...increases proportionately as the degree of overlap between the user's and the expert system's problem-solving processes decreases; that is, with decreasing cognitive consistency," (Lehner and Zirk, 1987).

"The user must have an accurate model of how that machine operates," (Lehner and Zirk, 1987). 


\section{Questions}

- When should we provide computerized decisions aids?

- How should these aids function?

- How should the computer's functioning be represented in the displays and controls that the user interacts with?

\section{Goal}

- To study possible answers to these questions in the context of en route flight planning. 


\section{Context: En route Flight Planning}

- Planning must take into consideration multiple competing and/or complementary goals (Wilensky, 1983).

- Decisions must be made in an information rich environment (Rouse, 1983).

- The flight crew and the computer must share data and inferences with each other.

- Such planning involves decision making under uncertainty.

- Decision making is really a group activity, involving ATC and Dispatch as well.

\section{GOALS}

*Study issues in the design of cooperative problemsolving systems

* Develop and evaluate design concepts for aiding real-time planning of flights 


\section{Approach}

\section{*Study human performance in existing environments}

*Build a test-bed for empirically studying alternative design concepts and principles (parttask simulation)

\section{*Evaluate promising concepts in full-task simulations}

\section{Flight Planning Testbed}

* Calculation of optimal altitudes

* Feedback on the implications of a plan

* Ability to explore "what-if" questions

* Spreadsheet-like computations and displays

* Integration of text and graphics displays

* Graphics-based exploration of flight plans

* Easy text-based editing of plans

* Alerting functions

* Accurate map projections for the whole world

* Shared plan generation 


\section{Flight Planning Testbed}

* For studying flight crews and dispatchers

* Part-Task Simulation

* Mac II

* Up to 6 Color Monitors

* Mouse and Keyboard Enry

* Real-Time and Simulation-Time Clocks

* Updating of Weather and Airport Statuses Over

* Automatic Recording of all Actions for Replay or Computer Analysis

* Trend Information

\section{Design Concepts}

* Personalized displays to accommodate particular circumstances and preferences

* Carefully designed functional groupings

(visual displays, menus, text displays)

* Compact displays

* Alternative methods of interaction (direct manipulation with mouse or trackball vs. keyboard entry)

* Develop intelligent "alarms" to focus attention on critical data and inferences (allow the pilot to "alarm" the computer as well?) 


\section{Design Concepts}

* Monitor for clearly questionable plans

(a critiquing system)

* Allow the pilot and the computer to exchange hypotheses, data, and inferences

* Take advantage of graphics-based planning aids to provide perceptual enhancement of problem solving

(Larkin and Simon, 1987)

* Design cooperative problem-solving systems rather than "autonomous" expert systems

* Allow pilots to ask "what if" questions

* To make it easy to ask "what if" questions, structure the architecture of the cooperative system around Sacerdoti's notion of an abstraction hierarchy

* To make it easy to ask "what if" questions, have the system infer the intentions of the pilot 


\section{Summary}

- Testbed

- Initial design concepts and implementations

- Methods for studying alternative designs 


\title{
PROGRAM ELEMENT III
}

\author{
ATC AUTOMATION AND \\ A/C-ATC INTEGRATION
}


